\title{
Readmission Rates Associated with Pharmacist Involvement in a Geriatric Transitional Care Management Clinic
}

\author{
E. Jared McPhail, PharmD; Vincent D. Marshall, MS; Tami L. Remington, PharmD; Sarah E. Vordenberg, PharmD, MPH, BCACP \\ University of Michigan College of Pharmacy
}

\begin{abstract}
Objective: To evaluate the impact of a post-discharge pharmacist telephone call on 30-and 90-day readmission rates as part of a transitional care management (TCM) service in a geriatric patient-centered medical home (PCMH).

Methods: Adults 60 years of age and older who had established primary care at the PCMH for at least one year and were discharged from the hospital between $7 / 1 / 2013$ and 2/21/2016 were included. Readmission rates for patients who received and did not receive a pharmacist TCM phone call were compared. Secondary data analysis was conducted between individuals who received all three components of the service compared with those who received on a nurse navigator plus primary care provider (PCP) visit.

Results: Among 513 discharges of unique patients (mean age, 80.4 years; women 63\%), 269 (52.4\%) received a pharmacist phone call. Readmission rates at 30 days were $8.9 \%$ for patients who received a pharmacist TCM phone call compared to $12.7 \%$ for those who did not receive this service (OR 0.67 [95\% Cl, 0.38-1.18; $P=0.17]$ ). When comparing only those individuals who received all three components of the service (pharmacist, nurse navigator, and $P C P)(n=215)$ compared to those who received only a nurse navigator plus $P C P$ visit $(n=66)$, there was no difference in 30-day readmission rates (7.9\% vs. 10.6\%, $p=0.49)$. However, there were significantly fewer readmissions within 90 -days $(16.3 \%$ vs. $31.8 \%, p=0.01)$.

Conclusion: Pharmacist phone calls as part of an interdisciplinary TCM service did not result in a statistically significant difference regarding readmission rates at 30 days; however, patients who received all three components of the service had significantly fewer readmissions at 90 days, compared to patients who did not speak with a pharmacist but did complete a visit with a nurse navigator and physician. Future research is needed to determine which patients may benefit the most from this service and to identify strategies to increase patient participation.
\end{abstract}

Keywords: geriatric, interdisciplinary, patient centered medical home, pharmacist, transitional care management

\section{Background}

The implementation of the Hospital Readmissions Reduction Program resulted in the development of a variety of transitional care management (TCM) programs aimed at decreasing hospital readmission rates. ${ }^{1-5}$ Hospitalizations have been associated with poor health outcomes for patients such as increased risk of hospital-acquired infections and medication errors. ${ }^{6}$ Additionally, it leads to increased cost for patients and health-systems. ${ }^{7}$ Nearly $19 \%$ of Medicare beneficiaries who are hospitalized are readmitted within 30 days, resulting in a \$17 billion cost annually. ${ }^{8}$

Patient centered medical homes (PCMH) are team-based, and utilize the strengths of multiple professions to provide patients with access to primary care, medication management, and social services. ${ }^{9}$ The $\mathrm{PCMHs}$ benefits both patients and stakeholders by being designed to accomplish the triple aim, including improvement of the patient experience of care, improving the health of populations, and reducing the per

Corresponding author: Sarah E. Vordenberg, PharmD, MPH, BCACP, Clinical Associate Professor

University of Michigan College of Pharmacy

428 Church St, Suite 3563 NUB

Ann Arbor, MI 48109

P: 734.763.6691; E: skelling@med.umich.edu capita cost of health care ${ }^{10}$ As part of a PCMH, pharmacists may provide medication management, patient education, side effect monitoring, medication overuse monitoring, development of personalized medication plans, and provision of TCM services. ${ }^{9}$ An effective pharmacist TCM intervention goes beyond medication reconciliation and is interdisciplinary and includes a clinical medication review and patient counseling. ${ }^{11}$ For example, in one $\mathrm{PCMH}$ program, pharmacist involvement was associated with significantly lower readmission rates $(7.7 \%$ vs $18.8 \%$; $\mathrm{P}=0.04) .{ }^{12}$ In this study, the patient arrived to clinic about 50 minutes before their scheduled PCP follow up appointment. The patient met with a licensed clinical social work (LCSW) and clinical pharmacist practitioner (CPP). The LCSW, CPP, and PCP had a 5-minute team huddle before the PCP completed the visit.

Telephone calls to patients have been explored as one strategy to increase the feasibility of incorporating pharmacists into TCM services. ${ }^{13,14}$ Several studies have demonstrated that a post-discharge follow-up phone call conducted by a pharmacist during the TCM process is associated with significant reductions in 30-day hospital readmissions. ${ }^{15-17}$ In a meta-analysis ( $\mathrm{n}=$ 32,538 ) in which the majority of studies utilized a telephone call as the method of contact, there was a significant reduction in the odds of all-cause 30-day readmission by $32 \%$ for patients receiving the pharmacy intervention compared to usual care, and there were no statistically significant differences when comparing telephone versus clinic visits. ${ }^{18}$ 
We conducted a previous study to determine whether an interdisciplinary TCM service reduced 30-day readmission rates among adults 60 years and older at a geriatric PCMH. ${ }^{19}$ The intervention included phone calls from a pharmacist and a nurse navigator followed by a clinic visit with a physician or nurse practitioner. The 30-day readmission rates were significantly lower among patients who received all components of the intervention compared to those who received usual care at sites with no TCM services (12.8\% vs. $7.9 \%, p=0.042$ ). This study expands on the work of our prior study by evaluating the impact of the pharmacist component of the intervention on 30- and 90-day readmission rates.

\section{Objective}

To evaluate the impact of a post-discharge pharmacist telephone call on 30- and 90-day all-cause readmission rates as part of an interdisciplinary TCM service in a geriatric PCMH as part of an academic health system.

\section{Methods}

\section{Program Description}

The TCM program is more fully described elsewhere, but in summary, it included a phone call from a nurse navigator within 2 days of hospital discharge, a phone call from a pharmacist 214 days after discharge, and a visit with a physician or nurse practitioner within 14 days after hospital discharge. ${ }^{19}$ The nurse navigator contacted the patient to establish willingness to receive care through the program, and triaged any minor medical concerns that occurred since discharge. During a 30minute appointment, the pharmacist assessed medical stability, reconciled medications, and performed a comprehensive medication review through a telephone intervention. Pharmacists also provided medication-related recommendations to the physician or nurse practitioner primarily via the electronic health record. Student pharmacists and pharmacy residents who participated in this program did so under the supervision of clinic pharmacists. It was the intent of the program that all patients would receive care from a nurse navigator, pharmacist, and physician or nurse practitioner, regardless of factors such as reason for the hospitalization, duration of hospitalization, or number of medications.

\section{Study Design}

Patients 60 years and older with established primary care at the geriatric PCMH for at least one year and who were hospitalized between 7/1/2013 and 2/22/2016 at the affiliated institution were included in this retrospective study. Patients who had planned hospitalizations, were discharged to another institution, or had frequent hospitalizations, defined as above the $99.99 \%$ percentile ( $n=2$, with 30 and 59 readmissions each), were excluded. The dates for this study were selected to prevent overlap with other institutional initiatives related to TCM services. Patients in the intervention group received a telephone call from a pharmacist (with or without contact from a nurse navigator and/or physician) and were compared to control patients who did not receive a call from a pharmacist [nurse navigator and/or primary care provider (PCP) contact without pharmacist contact]. The primary outcome of this study was all-cause 30- and 90-day hospital readmissions. A secondary analysis was a comparison of patients who received all three components of the service (nurse navigator telephone call, pharmacist telephone call, and PCP visit) with patients who received a nurse navigator call and PCP visit with no pharmacist telephone call. The study was approved by the University of Michigan Institutional Review Board.

\section{Data Collection and Analysis}

Data was collected via a custom data request from the University of Michigan Data Office for Clinical \& Translational Research and via manual data collection from the electronic health record, Epic (known within this particular institution as MiChart). Patient information related to demographics, index admission, rehospitalization, and outreach completion through various providers within the TCM service was collected electronically. Data regarding contact by the nurse navigator was collected manually. The accuracy of our data was confirmed with a manual chart review with a sample of patients demonstrating a $<5 \%$ error rate. Subject identifiers were coded and removed from the final data set. All data files were stored in an encrypted, password-protected file on a secure computer requiring username and password to access. Odds ratio was utilized to compare readmission rates between the patients that received the pharmacist telephone intervention (intervention group) and those who did not (control group) as well as patients who received all components of the intervention and only the nurse navigator and PCP visit (i.e. no pharmacist visit). We used an alpha of 0.05 , with no post-hoc multiple comparisons. We did not conduct a power calculation as this was secondary data analysis.

\section{Results}

A total of 513 patients were included in the study. The average age was 80.4 years (standard deviation $=8.2$ ), $63 \%$ were female, and $78 \%$ were white (table 1). While all components of the program were offered to patients, they engaged in varying combinations of services (table 2). Readmission rates at 30 days were $8.9 \%$ for patients who received a pharmacist TCM phone call compared to $12.7 \%$ for those who did not receive this service (OR 0.67 [95\% Cl, 0.38-1.18; $\mathrm{P}=0.17$ ]) (table 3).

When comparing patients who received all three components of the service $(n=216)$ with those who receive the nurse navigator and physician or nurse practitioner visit but no pharmacist visit ( $n=66)$, there was no significant difference in 30 -day hospital readmission rates $(7.9 \%$ vs. $10.6 \%, p=0.49)$ (table 4). However, there were significantly fewer readmissions within 90-days among patients who engaged with a pharmacist compared to those who received the other two components of the service $(16.3 \%$ vs. $31.8 \%, p=0.01)$.

\section{Discussion}

In this study, patients who participated in the pharmacist intervention as part of an interdisciplinary geriatric $\mathrm{PCMH}$ clinic 
did not have lower 30 day hospital readmission rates. However, in a secondary analysis, patients who completed all three aspects of the program had lower 90-day hospital readmission rates than those who only had the PCP and nurse navigator components.

A growing body of literature describes pharmacist participation in TCM program in order to reduce hospital readmissions. ${ }^{2-}$ 4,11,15-30 Roles of pharmacists in our study align were similar to those reported elsewhere - patient counseling, ${ }^{20,21}$ medication reconciliation, ${ }^{22,28}$ and medication management. ${ }^{30}$ While our study took place in an academic medical center $\mathrm{PCMH}$, other settings for TCM services include community pharmacies, ${ }^{5,26,27}$ home-based care, ${ }^{24,26}$ and through health insurance programs. ${ }^{4,5}$ Additional research is needed to understand the opportunities and barriers that exist in each of these diverse settings. For example, factors that likely benefited our program included the ability to access and document in the EHR as well as relationships with the care team. In contrast, we did not have access to medication dispensing records and frequently did not have existing relationships with the patients as patients generally interacted with the pharmacists only for TCM services.

In a systematic review, Hansen et al. reported that interventions to reduce 30-day hospital readmissions could be categorized into three domains - predischarge interventions, postdischarge interventions, and bridging interventions. ${ }^{13}$ While we studied a postdischarge intervention, it is important to acknowledge that predischarge interventions also occurred. For example, before a patient was discharged from the hospital, they their received discharge planning and an appointment was scheduled. Similar to the results in this review, our study focused on a quality improvement initiative at one institution. However, we did attempt to assess the impact of the pharmacist intervention, as opposed to only evaluating the entire TCM bundle.

A significant limitation in this study was that approximately one-half of patients who were eligible for pharmacist services did not receive them. While the causes were not formally evaluated, observations from clinical practice include patients not expecting a phone call due to poor communication or receiving an overwhelming amount of information at discharge, outdated patient telephone numbers in the electronic health record, patient disinterest or forgetfulness, readmission occurring before the scheduled pharmacist intervention, lack of pharmacist availability, or patient refusal. Furthermore, our study was a secondary analysis of a larger study. It is possible that we had significant findings regarding 90-day readmissions by chance given the number of analyses that have been conducted. Finally, patient who did not to participate in the pharmacist visit may have different clinical or social characteristics compared to those who did participate in the program and this could have resulted in their higher readmission rates.
Additional research is needed to determine which patients may benefit most from TCM services and to identify strategies to increase patient engagement. Studies of telephone-based pharmacist interventions conducted in the future may benefit from a more thorough phone call scheduling process, confirmation of patient willingness to participate, and education regarding the purpose and potential benefit of the pharmacist intervention.

\section{Conclusion}

Pharmacist phone calls as part of an interdisciplinary TCM service did not result in a statistically significant difference 30day readmission rates. However, we found that patients who received all components of the program had lower 90-day hospital readmission rates compared to those who received only the PCP and nurse navigator components. Future research is needed to determine which patients may benefit the most from this service and to identify strategies to increase patient participation.

\section{Acknowledgements: None}

Conflicts of interest: We declare no conflicts of interest or financial interests that the authors or members of their immediate families have in any product or service discussed in the manuscript, including grants (pending or received), employment, gifts, stock holdings or options, honoraria, consultancies, expert testimony, patents and royalties.

Funding: The American College of Clinical Pharmacy Ambulatory Care Practice Based Research Network Seed Grant funded a portion of this study.

Previous presentations of the work: American Society of Health-System Pharmacists Midyear Meeting, Orlando, FL, 2017

\section{References}

1. Center for Medicare and Medicaid Services. Readmissions Reduction Program (HRRP). Available at http://www.cms.gov/Medicare/Medicare-Fee-ForService-Payment/AcutelnpatientPPS/ReadmissionsReduction-Program.html. Accessed January 7, 2019.

2. Luder H, Zavadsky A, Bukowitz A, Baumann A, Boylan PM, Croley KS, Fu Denise, Kelling SE, Winder M, Register D. Transitions of care: Case examples resources. American Pharmacists Association. June 2017.

3. Stranges PM, Marshall VD, Walker PC et al. An Interdisciplinary Intervention for Reducing Readmission among Older Adults in a Patient-Centered Medical Home. Am J Manag Care. 2015;21(2):106-113.

4. Polinksi JM, Moore JM, Kyrychenko P et al. An Insurer's Care Transition Program Emphasizes Medication Reconciliation, Reduces Readmissions And Costs. Health Aff. 2016;35(7):1222-1229. 
5. Kelling SE, Bright DR, Ulbrich TR, et al. Development and implementation of a community pharmacy medication therapy management-based transition of care program in the managed Medicaid population. Innov Pharm. 2013;4(4):Article 137.

6. Centers for Disease Control and Prevention. 2016 National and State Healthcare-Associated Infections Progress Report. Available at www.cdc.gov/hai/progress-report/index.html. Accessed January 7, 2019.

7. Choi I, Lee SM, Flynn L, et al. Incidence and treatment costs attributable to medication errors in hospitalized patients. Res Social Adm Pharm. 2016;12(3):428-37.

8. Center for Medicare and Medicaid Services. CMS Quality Strategy. Available at https://www.cms.gov/Medicare/Quality-InitiativesPatient-AssessmentInstruments/QualityInitiativesGenInfo/Downloads/CMS -Quality-Strategy.pdf. Accessed January 7, 2019.

9. Taylor ER, Lake T, Nysenbaum J, et al. Coordinating care in the medical neighborhood critical components and available mechanisms. White Paper. Rockville, MD: Agency for Healthcare Research and Quality. June 2011.

10. Peikes D, Zutshi A, Genevro et al. Early evaluations of the medical home: building on a promising start. Am J Manag Care. 2012;18(2):105-116.

11. Ensing HT, Stuijt CC, van den Bemt BJ, et al. Identifying the optimal role for pharmacists in care transitions: a systematic review. J Manag Care Spec Pharm. 2015;21(8):614-36.

12. Hawes EM, Smith JN, Pinelli NR et al. Accountable Care in Transitions (ACTion): A Team-Based Approach to Reducing Hospital Utilization in a Patient-Centered Medical Home. J Pharm Pract. 2018;31(2):175-82.

13. Hansen LO, Young RS, Hinami K, et al. Interventions to reduce 30-day rehospitalization: a systematic review. Ann Internal Medicine 2011;155(8):520-528.

14. Hesselink G, Schoonhoven L, Barach P, et al. Improving patient handovers from hospital to primary CareA systematic review. Ann Internal Medicine. 2012;157(6):417-428.

15. Kirkham HS, Clark BL, Paynter J, et al. The effect of a collaborative pharmacist-hospital care transition program on the likelihood of 30-day readmission. Am J Health Syst Pharm. 2014;71(9):739-745.

16. Ploenzke Chris, Kemp T, Naidl T, et al. Design and implementation of a targeted approach for pharmacistmediated medication management at care transitions. J Am Pharm Assoc. 2016:56(3):303-309.

17. Sanchez GM, Douglass MA, Mancuso MA. Revisiting Project Re-Engineered Discharge (RED): The Impact of a Pharmacist Telephone Intervention on Hospital Readmission Rates. Pharmacotherapy. 2015;35(9):80512.
18. Rodrigues $C R$, Harrington AR, Murdock $N$ et al. Effect of Pharmacy-Supported Transition-of Care Interventions on 30-Day Readmissions: A Systematic Review and Meta-analysis. Ann Pharmacother. 2017;51(10):866889.

19. Farhat NM, Vordenberg SE, Marshall VD, et al. Evaluation of interdisciplinary geriatric transitions of care on readmission rates. Am J Manag Care. 2019;25(7):e219-223.

20. Schnipper JL, Kirwin JL, Cotugno MC, et al. Role of pharmacist counseling in preventing adverse drug events after hospitalization. Arch Intern Med. 2006;166(5):565-571.

21. Shah M, Norwood CA, Farias S, et al. Diabetes transitional care from inpatient to outpatient setting: Pharmacist discharge counseling. J Pharm Pract. 2012;26(2):120-124.

22. Kilcup M, Schultz D, Carlson J, Wilson B. Postdischarge pharmacist medication reconciliation: impact on readmission rates and financial savings. J Am Pharm Assoc. 2013;53(1):78-84.

23. Anderson SL, Maars JC, Vande Griend JP, Hanratty R. Implementation of clinical pharmacy specialistmanaged telephonic hospital discharge follow-up program in a patient centered medical home. Popul Health Manag. 2013;16(4):235-241.

24. Pherson EC, Shermock KM, Efird LE, et al. Development and implementation of a postdischarge home-based medication management service. Am J Health Syst Pharm. 2014;71(18):1576-1583.

25. Arnold ME, Buys L, Fullas F. Impact of pharmacist intervention in conjunction with outpatient physician follow-up visits after hospital discharge on readmission rate. Am J Health Syst Pharm. 2015;72(11 Suppl 1):S3642.

26. Kalista T, Lemay V, Cohen L. Postdischarge community pharmacist-provided home services for patients after hospitalization for heart failure. J Am Pharm Assoc. 2015;55(4):438-442.

27. Luder HR, Frede SM, Kirby JA, et al. TransitionRx: Impact of community pharmacy postdischarge medication therapy management on hospital readmission rate. $J$ Am Pharm Assoc. 2015;55(3):246-254.

28. Phatak A, Prusi R, Ward B, et al. Impact of pharmacist involvement in the transitional care of high-risk patients through medication reconciliation, medication education, and postdischarge call-backs (IPITCH Study). J Hosp Med. 2016 Jan;11(1):39-44.

29. Ni W, Colayco D, Hashimoto J, et al. Impact of a pharmacy-based transitional care program on hospital readmissions. Am J Manag Care. 2017 Mar;23(3):170176.

30. Yang S. Impact of pharmacist-led medication management in care transitions. BMC Health Serv Res. 2017 Nov 13;17(1):722. 
Table 1. Demographic information for patients who received and did not receive pharmacist transitional care management services

\begin{tabular}{|ccc|}
\hline & $\begin{array}{c}\text { Control } \\
(\mathrm{n}=269)\end{array}$ & $\begin{array}{c}(\mathrm{n}=244) \\
80.0(8.5)\end{array}$ \\
\hline Age, mean (SD) & $80.8(8.0)$ & $151(61.9)$ \\
Females, $\mathrm{n}(\%)$ & $171(63.6)$ & $191(78.3)$ \\
White, $\mathrm{n}(\%)$ & $209(77.7)$ & $2.6(2.6)$ \\
\hline
\end{tabular}

Table 2. Number of participants by type of health care professional appointments

\begin{tabular}{|ll|}
\hline Health care professional & Number of patients (\%) \\
\hline Nurse navigator, Pharmacist, and PCPa & $215(41.9)$ \\
Nurse navigator and PCPa & $66(12.9)$ \\
Nurse navigator and Pharmacist & $30(5.8)$ \\
Nurse navigator only & $36(7.0)$ \\
PCPa only & $22(4.3)$ \\
Pharmacist and PCPa & $24(4.7)$ \\
\hline
\end{tabular}

a Primary care provider

Table 3. Number and percent of 30- and 90-day readmissions by participation in pharmacist component of transitional care management service

\begin{tabular}{|c|c|c|c|c|}
\hline & $\begin{array}{c}\text { Intervention }^{a} \\
(n=269) \\
n(\%)\end{array}$ & $\begin{array}{c}\text { Control }^{b} \\
(n=244) \\
n(\%)\end{array}$ & OR (95\% C.I.) & p-value \\
\hline \multicolumn{5}{|l|}{ 30-day } \\
\hline Readmission & $24(8.9)$ & $31(12.7)$ & \multirow{2}{*}{$0.67(0.38-1.18)$} & \multirow{2}{*}{0.17} \\
\hline No readmission & 245 (91.1) & $213(87.3)$ & & \\
\hline \multicolumn{5}{|l|}{ 90-day } \\
\hline Readmission & $47(17.5)$ & $59(24.2)$ & \multirow{2}{*}{$0.66(0.43-1.02)$} & \multirow{2}{*}{0.06} \\
\hline No readmission & $222(82.5)$ & $185(75.8)$ & & \\
\hline
\end{tabular}

a Participated in pharmacist transitional care management appointment

${ }^{b}$ Did not participate in pharmacist transitional care management appointment

${ }^{c}$ Odds ratio $p$-value

Table 4. Secondary data analysis of the number and percent of

30- and 90-day readmissions among patients who participated in all three components of the service compared to nurse navigator and primary care provider visits

\begin{tabular}{|c|c|c|c|c|}
\hline & $\begin{array}{c}\text { Intervention }^{a} \\
(n=215) \\
n(\%)\end{array}$ & $\begin{array}{c}\text { Control }^{b} \\
(n=66) \\
n(\%)\end{array}$ & OR (95\% C.I.) & $p$-value ${ }^{c}$ \\
\hline \multicolumn{5}{|l|}{ 30-day } \\
\hline Readmission & $17(7.9)$ & $7(10.6)$ & \multirow{2}{*}{$0.72(0.29-1.95)$} & \multirow{2}{*}{0.49} \\
\hline No readmission & $198(92.1)$ & $59(89.4)$ & & \\
\hline \multicolumn{5}{|l|}{ 90-day } \\
\hline Readmission & $35(16.3)$ & $21(31.8)$ & \multirow{2}{*}{$0.42(0.22-0.80)$} & \multirow{2}{*}{$<0.01$} \\
\hline No readmission & $180(83.7)$ & $45(68.2)$ & & \\
\hline
\end{tabular}

a Participated in pharmacist, nurse navigator, and primary care provider transitional care management appointments

${ }^{b}$ Participated in nurse navigator and primary care provider transitional care management appointments

${ }^{c}$ Odds ratio $p$-value 